If congruence axioms are present, a given line $a$ of one class may correspond to any line $a^{\prime}$ of the $\infty^{2}$ lines in the other class. A given point $A$ of $a$ may correspond to any one of the $\infty^{1}$ points of $a^{\prime}$. A line perpendicular to $a$ at $A$, however, must correspond to a line perpendicular to $a^{\prime}$ at $A^{\prime}$. The whole correspondence is therefore fixed when a unit of length $A^{\prime} B^{\prime}$ is chosen on $a^{\prime}$ to correspond to $A B$ on $a$. There are thus $\infty^{4}$ ways in which the correspondence between two planes defined by order and congruence axioms may be set up. If order axioms alone are used, it is clear that the number of correspondences must be the same as the number of collineations of a cartesian plane into itself leaving the line at infinity invariant, and this count is $\infty^{6}$. A system of axioms stated in terms of order and congruence combined is therefore more categorical than one in terms of order alone.

Oswald Veblen.

\title{
COLLEGE ALGFBRA.
}

A College Algebra. By Henry Burchard Fine. Ginn and Company, 1905. viii +595 pp.

Among all the text-books of college algebra, this book by Professor Fine, of Princeton, is distinguished by its broad grasp of the subject as a branch of mathematical science and by its entire freedom from the misleading statements and positive blunders which are all too common in our current text-books. What measure of success it will obtain in the class-room it would be hard to prophesy ; my own impression is that on account of the close logical interdependence of its various parts the book will be found less available for those classes which wish to take up only a few detached chapters on separate topics than for those classes which can go through the whole subject in a systematic manner. However this may be, it is certainly a book which every teacher of mathematics, whether he happens to have a class in algebra or not, should own and read. In fact, if a mathematical library were to contain only one English text-book in algebra (besides the two-volume treatise of Chrystal) I should unhesitatingly recommend the present book.

The book is divided into two parts: "a preliminary part devoted to the number system of algebra, and a principal part devoted to algebra itself." 
The first part (about one-seventh of the whole) contains a systematic treatment of the rational, irrational, and imaginary numbers, in the light of the researches of Dedekind and Cantor; these researches have been known on the continent for over thirty years, but are here for the first time made accessible in a satisfactory manner in an English text-book.* If there is anyone who feels that such discussions are unimportant, on the ground that the old-fashioned treatment of irrationals and imaginaries in our current algebras is well enough as it is, let him ask any freshman class in mathematics at the opening of the college year what meanings the words "irrational number" and "imaginary number" convey to them. If his experience is anything like my own, he will find that not a single member of the class has any clear-cut notion of these concepts. The replies obtained will be simply variations on the following, which are entirely typical of those I have received: "An irrational number is one which has no exact value ;" "An imaginary number is one which has no value at all and does not really exist." Interesting objects, indeed! And yet these are the mystical entities which the school boy is taught to add and subtract, to multiply and divide, as if they were ordinary numbers in the arithmetical sense. No wonder that an occasional bright pupil will protest that "you cannot extract the square root of a number which hasn't got any square root," and assure you that "to put a radical sign around a minus quantity always seemed like trying to squeeze money out of a man who didn't have any"! Such are the fruits of the present half-hearted treatment of these subjects in the ordinary text-books; any fresh and rigorous discussion, like that of Professor Fine's, should therefore be cordially welcomed by every lover of common sense and common honesty in teaching.

How much of this preliminary part will be available for the class-room, every teacher must decide for himself; the college student at any rate should be made to grasp at least the main principles which underlie the work, namely : (1) that the number system is merely a system of signs which we invent for our own convenience in handling certain practical problems (like the problems of counting and measuring); (2) that the "operations" on these numbers which are almost inevitably suggested by the

* Since this book was published, another excellent account of the Dedekind-Cantor theory has appeared in Pierpont's Theory of functions of real variables. 
exigencies of those problems are essentially defined by the fundamental laws which govern them (e.g., the commutative, associative, and distributive laws for addition and multiplication); and (3) that all the propositions of algebra are logically deducible from these fundamental laws without further reference to the nature of the numbers involved.

It will be seen that from this point of view the definitions of the numbers themselves are not important; the fundamental laws of operation are all that we need to know. In other words, any system which obeys the laws of rational algebra is a system of rational numbers; any system which obeys the laws of real algebra is a system of real numbers; etc.* To fix our ideas, however, and also to show that the laws are not inconsistent, it is desirable to exhibit some concrete example of such a system. The principal example which Professor Fine selects for this purpose (to speak only of the real numbers) is the one proposed by Dedekind and Cantor, as already mentioned; in this system, the rational numbers are pictured as pairs of integers and the real numbers as "separations" in the series of rationals (or as "regular sequences" of rationals). Later on another example is introduced, paralleling the first, in which the real numbers are pictured as points on a line (or better, as distances along a line). For the purposes of an elementary course it would have been preferable, I think, if the autbor had given the principal place to the second or more geometrical of these two parallel methods of representation. In all the formal processes of real algebra, it makes no difference whether the letters $a, b, x, y$, etc., stand for "separations in a series of pairs of integers" or for "distances along a line"; and surely the latter interpretation is more accessible to an elementary student, and much more useful in practice. The only advantage which the Dedekind-Cantor example possesses lies in the fact that it enables us to establish the consistency of the laws of real algebra on purely logical grounds, without any appeal to geometrical intuitions - considerations which may properly be postponed until a later stage in the student's mathematical study.

Turning now to the main part of the book, on algebra proper, we find a full discussion of "everything relating to algebra that

* Cf. Transactions Amer. Math. Society, vol. 6 (1905), pp. 209-229, especially $\& 10$. 
a student is likely to need during his school and college course." The only topic which one will seek in vain is the binary scale of notation, which the author would regard, I suppose, as belonging more properly in arithmetic. The treatment of the various topics is fresh and stimulating, and is well calculated to keep before the student, in their proper relations, the two essentials of a mastery of the subject, namely sound reasoning and skillful manipulation.

It is of course true that no human reasoning can be more than approximately rigorous, and that in elementary work it is not desirable to carry this approximation beyond the limits of understanding of the pupil; but surely some advance over our current text-books is to be songht. A good example of what seems to me a proper degree of rigor in an elementary text-book is the excellent discussion of the solution of equations on page 114 : "It is important to remember that the mere fact that a certain value of $x$ has been derived from an equation by the rules of reckoning does not prove it to be a root; the process must be reversible to warrant this conclusion." This is not a mere subtlety inserted for the sake of an appearance of rigor ; it is a bit of common sense which is absolutely indispensable even if we wish merely to "get the right answer." For example (page 288), in solving the simple irrational equation $x-7-\sqrt{x-5}=0$, we find that if $x$ is a root, then $x$ is 9 or 6 ; but it does not follow that (conversely) if $x$ is 9 or 6 , then $x$ is a root; as a matter of fact, the value 6 is not a root. There is no more reason for supposing that the converse of a true proposition is also true in algebra than there is for making the same mistake in geometry. It is to be regretted that the exercises on page 290 were not so chosen as to emphasize this point.

The early introduction of synthetic division and of the method of undetermined coefficients is to be commended, as is also the use of graphs in connection with equations of the first and second degrees ; in this graphical discussion, however, it might have been well to point out the sharp distinction between a curve as the picture of a function of one variable and a surface as the picture of a function of two variables.

The chapter on simultaneous quadratics, while rather diffi. cult, will well repay a careful study, since it replaces the usual list of special devices by a connected treatment of the subject as a logical whole. It should be noticed, however, that equations like $x^{2}+3 y^{2}=31,7 x^{2}-2 y^{2}=10$ (page 324) should 
be regarded not as quadratic equations in $x$ and $y$ but as linear equations in $x^{2}$ and $y^{2}$; and the use of infinite solutions is of doubtful value.

In the chapter on the theory of equations it would have been well to give a few examples of numerical equations with irrational coefficients, since the equations which come up in practice are frequently of this type. Again, in the chapter on logarithms, the awkward double-headed rule for finding the characteristic which is now in common use might well have been replaced by the older and simpler rule which refers to the units' place instead of to the decimal point.

The later chapters of the book are a veritable store-house of accurate and well-selected information concerning infinite series, infinite products, continued fractions, etc. Special attention should be called to the last chapter of all, which contains a brief but admirable discussion of the continuity of functions of one and two variables, including correct definitions of such terms as upper and lower limit and oscillation of a function, a proof of Weierstrass's theorem that a continuous function actually reaches both its upper and its lower limit in any closed interval, and Argand's proof of the fundamental theorem of algebra. This chapter is of course not intended for a first-year class, but will be valuable for reference in the later years of the student's course.

It may be noted in conclusion that the book has an excellent index, and that answers to the exercises will be supplied by the publishers on the request of a teacher.

E. V. Huntington.

\section{FREUND'S TRANSLATION OF BALL'S HISTORY OF MATHEMATICS.}

Histoire des Mathématiques. Par W. W. Rouse Ball. Edition française revue et augmentée. Traduite sur la troisième édition anglaise par L. Freund. Tome premier. Paris, A. Hermann, 1906 . viii $+422 \mathrm{pp}$.

IT is an interesting fact that France, which produced in Paul Tannery one of the greatest, if not the greatest historian of mathematics of our time, has not for a hundred years produced a great general history of the subject. Libri was a scholarly 